1986

\title{
Anisotropy of critical correlations in moderately delocalized cerium and actinide systems
}

Nicholas Kioussis

Bernard R. Cooper

Follow this and additional works at: https://researchrepository.wvu.edu/faculty_publications

\section{Digital Commons Citation}

Kioussis, Nicholas and Cooper, Bernard R., "Anisotropy of critical correlations in moderately delocalized cerium and actinide systems" (1986). Faculty Scholarship. 56.

https://researchrepository.wvu.edu/faculty_publications/56

This Article is brought to you for free and open access by The Research Repository @ WVU. It has been accepted for inclusion in Faculty Scholarship by an authorized administrator of The Research Repository@ WVU. For more information, please contact ian.harmon@mail.wvu.edu. 


\title{
Anisotropy of critical correlations in moderately delocalized cerium and actinide systems
}

\author{
Nicholas Kioussis and Bernard R. Cooper \\ Department of Physics, West Virginia University, Morgantown, West Virginia 26506
}

(Received 19 November 1985)

\begin{abstract}
The equilibrium and excitation magnetic behavior of a class of cerium and light actinide compounds have been explained previously, in a theory first developed by Siemann and Cooper, in terms of a band-f-electron anisotropic hybridization-mediated two-ion interaction of the CoqblinSchrieffer type. Using the same theory, we present here a calculation, within the random-phase approximation, of the longitudinal component of the static wave-vector-dependent susceptibility in the paramagnetic phase. The calculations have been performed in the presence of a cubic crystal field (CF) and yield results for the ratio of inverse critical correlation lengths, $\kappa_{\|} / \kappa_{\perp}$, parallel and perpendicular to the moment direction, that compare well with those of diffuse critical neutron scattering experiments. In $\mathrm{Ce}^{3+}\left(f^{1}\right)$ compounds, we find that as the CF interaction ( $\Gamma_{7}$ ground state) predominates over the two-ion interaction, the relative strength of the coupling within the ferromagnetic $\{001\}$ planes (with moments perpendicular to the planes) and that between the $\{001\}$ planes is gradually reversed, resulting in a ratio $\kappa_{\|} / \kappa_{\perp}$ smaller than unity, as is experimentally observed. We also present results for the effect of differing intraionic $(L-S$, intermediate, and $j-j)$ coupling on $\kappa_{\|} / \kappa_{1}$ for the case of $\mathrm{Pu}^{3+}\left(f^{5}\right)$ and $\mathrm{U}^{3+}\left(f^{3}\right)$ compounds.
\end{abstract}

\section{INTRODUCTION}

The heavier $\mathrm{NaCl}$-structure monopnictides of cerium and the light actinides ( $\mathrm{U}, \mathrm{Np}$, and $\mathrm{Pu}$ ) exhibit ${ }^{1-4}$ unusual magnetic structures, and the cerium ${ }^{1}$ and plutonium ${ }^{3,4}$ compounds show extremely large anisotropy with strong preference for moment alignment along the cube edge. $\mathrm{CeBi}$ and the uranium monopnictides except for USb, i.e., $\mathrm{U} X(X=\mathrm{N}, \mathrm{P}, \mathrm{As}, \mathrm{Bi})$, order initially in a collinear type-I antiferromagnetic (AF-I) structure with ferromagnetic $\{001\}$ planes stacked in a $(+-)$ sequence and moments perpendicular to the planes. ${ }^{2}$ The Néel temperature $\left(T_{N}\right)$ is $25 \mathrm{~K}$ in $\mathrm{CeBi}$, and $T_{N}$ steadily increases ${ }^{2}$ from $53 \mathrm{~K}$ in $\mathrm{UN}$ to $285 \mathrm{~K}$ in UBi. USb orders at $241 \mathrm{~K}$ with a noncollinear (triple- $k$ ) type-I structure. ${ }^{2}$ In $\mathrm{CeSb}$, although the moment fluctuations above the Neel temperature $(16 \mathrm{~K})$ are of ${ }^{5}$ AF-I type, the long-range ordering at $T_{N}$ is $^{6}$ unusual with $\{001\}$ planes alternating in $(+-0)$ sequence. PuSb undergoes ${ }^{3,4}$ an initial ordering to a longperiod (about ten-layer spacings) AF phase at $85 \mathrm{~K}$ with modulation wave vector along the [001] moment direction, and undergoes a first-order transition to a ferromagnetic phase at $75 \mathrm{~K}$. The nature of the magnetic structure of CeAs and CeP has been ${ }^{2}$ controversial. Hälg et al. ${ }^{7}$ upon applying a magnetic field pointed out that the anisotropic behavior of the transverse modes of the excitation spectrum in CeAs provides evidence of a single- $\mathbf{k}$ structure. Burlet et al., ${ }^{8}$ on the other hand, conclude that the magnetic structure of CeAs is a triple-k structure, but that one can induce collinear antiferromagnetism with a relatively weak field of $17 \mathrm{kOe}$ or less.

Upon diluting the $\mathrm{Ce}$ with nonmagnetic $\mathrm{La}$ or $\mathrm{Y}$ in $\mathrm{CeBi}$ and $\mathrm{CeSb},{ }^{9}$ the easy axis of magnetization is observed to change from $\langle 001\rangle$ to $\langle 111\rangle$ at sufficiently low Ce concentrations, suggesting that the $\langle 001\rangle$ anisotropy arises from a two-ion exchange interaction mechanism be- tween the $\mathrm{Ce}^{3+}$ ions. The origin of this strong anisotropy was shown ${ }^{10}$ to arise predominantly from the resonant hybridization of moderately delocalized $f$ electrons with band electrons, i.e., a hybridization mechanism of the Coqblin-Schrieffer ${ }^{11}$ (CS) type. This hybridizationmediated interaction yields strong intraplanar coupling and a much weaker coupling between planes transverse to the moment direction. ${ }^{10}$

Neutron scattering ${ }^{12}$ gives direct and detailed information about the strong cubic anisotropy in these compounds. Diffuse critical neutron scattering (DCNS) experiments above the ordering temperature $T_{N}$ provide direct information concerning the short-range moment fluctuations, from which the size of the correlation length can be quantitatively determined, and the possible multicritical behavior of the system. Inelastic neutron scattering (INS) experiments, on the other hand, yield information about the long-range spin correlations and the spectrum of the transverse magnetic excitations of the system. DCNS experiments performed on cerium monopnictides $^{5,13,14}(\mathrm{CeBi}, \mathrm{CeSb}$, and CeAs) and uranium monopnictides ${ }^{15-17}$ (USb, UAs, and UN) show that the critical scattering above $T_{N}$ is essentially longitudinal with no evidence of a transverse component; thus, the static wave-vector-dependent longitudinal component of the susceptibility $\chi^{z z}(\mathbf{q})$ is much greater than the corresponding $\chi^{x x}(q)$ and $\chi^{y y}(q)$ components for $q$ along the [001] direction. Furthermore, the critical scattering is highly anisotropic in reciprocal space. For $\mathrm{CeBi}, \mathrm{CeSb}$, and the uranium monopnictides, the constant-intensity contours have the form of an ellipsoid of revolution with its long axis along the [001] direction (cigarlike shape), suggesting that the moments are attempting to order in a two-dimensional fashion in $\{001\}$ sheets and the correlation range within planes is larger than that between planes. In the case of CeAs, this relative strength of intra- and interplanar cou- 
pling is reversed, and the scattering has the form of a lenslike shape in reciprocal space, with the short axis parallel to the moment direction. The ratio, $\kappa_{\|} / \kappa_{1}$, of the inverse correlation lengths parallel and perpendicular to the moment direction has been found to be approximately independent of temperature and assumes the values ${ }^{12}$ of about 5.0 in USb, 3.8 in UAs, 2.8 in UN, 2.5 in $\mathrm{CeBi}, 1.8$ in $\mathrm{CeSb}$, and 0.6 in CeAs.

In the cerium monopnictides the octahedral crystal field splits the $\mathrm{Ce}^{3+}$ free-ion ground-state $J=\frac{5}{2}$ multiplet into a ground-state $\Gamma_{7}$ doublet and an excited $\Gamma_{8}$ quartet. In CeAs, the crystal-field (CF) splitting in the paramagnetic phase is about $160 \mathrm{~K}$ (approximately half of its expected $^{18}$ value upon extrapolation from the heavier rareearth monopnictides) and is much larger than the Néel temperature of $8 \mathrm{~K}$. In CeAs, the $\mathrm{CF}$ interaction presumably dominates the hybridization-mediated two-ion interaction, resulting in a strong quenching of the free-ion moment $\left(2.1 \mu_{B}\right)$ to about one third of its value. ${ }^{12}$ On the other hand, $\mathrm{CeSb}$ and $\mathrm{CeBi}$ have anomalously small $\mathrm{CF}$ splittings in the paramagnetic phase, of about 37 and $8 \mathrm{~K}$, compared to the "bare" value of about $250 \mathrm{~K}$ expected upon extrapolation from the heavier rare-earth antimonides and bismuthides. ${ }^{18}$ This reduction has been explained on the basis of hybridization effects ${ }^{19-21}$ between $p$ - and $d$-band states and the quasilocalized $f$ states. High-temperature susceptibility measurements ${ }^{22}$ on UP and UAs and elastic neutron scattering experiments ${ }^{23}$ on USb have indicated the existence of significant CF splittings in the paramagnetic phase for the $\mathrm{U}^{3+}\left(f^{3}\right)$ compounds.

The static and dynamic magnetic properties of the heavier cerium monopnictides ( $\mathrm{CeBi}$ and $\mathrm{CeSb}$ ) have been successfully explained ${ }^{24-26}$ using the hybridizationmediated two-ion interaction. Using the same interaction we present here a random-phase approximation (RPA) calculation of the longitudinal component of the static wave-vector-dependent susceptibility, $\chi^{z z}(\mathbf{q})$, in the paramagnetic phase. In our calculations we have empirically treated the anomalous CF effects by introducing a phenomenological CF parameter with a CF splitting equal to the observed one. Such a simple treatment neglects the temperature dependence ${ }^{19,20}$ of the hybridization-induced $C F$ effect. Using ferromagnetic nearest-neighbor $\left(E_{1}\right)$ and second nearest-neighbor $\left(E_{2}\right)$ hybridization-mediated two-ion interactions (in the notation of Refs. 10 and 24-26), and a small antiferromagnetic third nearestneighbor $\left(E_{3}\right)$ interaction to stabilize the AF-I phase, we have calculated the ratio of the inverse correlation lengths, $\kappa_{\|} / \kappa_{1}$, in the vicinity of the Néel temperature. This ratio is found to be approximately temperature independent. Our numerical results are in close agreement with those of DCNS experiments, ${ }^{12}$ thus further substantiating the experimental relevance of the hybridization-mediated interaction in this family of compounds. We find $\kappa_{\|} / \kappa_{\perp}$ to be about 2 for small CF splitting, showing that the magnetic interactions are stronger within the ferromagnetic planes than the interplanar couplings. However, as the CF interaction ( $\Gamma_{7}$ ground state) predominates over the hybridization-mediated two-ion interaction, we find that the relative strength of intra- and interplanar coupling is gradually reversed, resulting in a ratio $\kappa_{\|} / \kappa_{\perp}$ smaller than unity.

The theory of hybridization-mediated anisotropic twoion interaction has been extended ${ }^{4,27-30}$ to the general case of $f^{n}$ ions. The angular dependence of the interaction in this theory depends on the $f$ occupation number and on the nature of intraionic coupling. Using the same model interaction, we have also investigated the effect of intraionic coupling on the size of $\kappa_{\|} / \kappa_{\perp}$ for the case of $\mathrm{Pu}^{3+}\left(f^{5}\right)$ compounds such as $\mathbf{P u S b}$, and for the case of $\mathrm{U}^{3+}\left(f^{3}\right)$ compounds with the behavior of UP, UAs, and $\mathrm{USb}$ in mind. Including the possibility of long-period AF structures in the case of $\mathrm{Pu}^{3+}$ systems, we find numerical results for $\kappa_{\|} / \kappa_{\perp}$ which are almost identical for both the $L-S$ and intermediate coupling (IC) cases. This is not surprising, since the IC ground state of the $\mathrm{Pu}^{3+}$ ion is composed primarily ( $\simeq 75 \%$ ) of the ${ }^{6} \mathrm{H}$ state (which is the ground state in the $L-S$ coupling limit) and four ${ }^{4} G$ states ( $\simeq 25 \%$ ). More importantly, for the case of longitudinal polarization of the moment with respect to the modulation direction (as observed ${ }^{3}$ experimentally) we find an imaginary $\kappa_{\|}$. This result is consistent with those of the magnetization calculations ${ }^{29}$ which predict a transverse polarization with moments aligned along the cube edge.

For the case of transverse polarization we find $\kappa_{\|} / \kappa_{\perp}$ to be smaller than unity for both $L-S$ and intermediate coupling, and for a wide range of $E_{2} / E_{1}, E_{3} / E_{1}$, and of the CF splitting. On the other hand, on going to the $j-j$ coupling limit, the ratio $\kappa_{\|} / \kappa_{\perp}$ changes dramatically; in this limit the angular dependence of the interaction for $\mathrm{Pu}^{3+}$ becomes ${ }^{28}$ ceriumlike resulting in a ratio $\kappa_{\|} / \kappa_{1}$ of the order or greater than 2. The $\mathrm{U}^{3+}$ ion, pertinent to the case of the uranium monopnictides, shows behavior close to the $L-S$ coupled limit for the $f^{3}$ system. ${ }^{22,23}$ In contrast to the $\mathrm{Pu}^{3+}$ case, we find $\kappa_{\|} / \kappa_{\perp}$ to be greater than unity (in the presence or absence of CF interactions) for the AF-I structure in both the $L-S$ and the less realistic $j-j$ intraionic coupling limits, in qualitative agreement with DCNS experiments. ${ }^{12}$ This different behavior of $\kappa_{\|} / \kappa_{\perp}$ in the $f^{3}$ and $f^{5}$ systems arises from the different angular dependence of the exchange coefficients in these two systems.

The effects associated with band- $f$ hybridization have led to an understanding of the equilibrium and excitedstate magnetic behavior of cerium and light actinide systems with magnetic behavior dominated by hybridization-mediated interactions ${ }^{31}$ and to an understanding of the related phenomenon of hybridization dressing of the crystal field. ${ }^{20}$ The present work further substantiates the experimental relevance of the hybridization-induced interactions through using them to understand near-critical-point anisotropic correlation length behavior as observed by use of diffuse neutron scattering. In Sec. II we present the formalism for calculating, within the RPA, the longitudinal component of the static susceptibility $\chi^{z z}(q)$, and the ratio $\kappa_{\|} / \kappa_{\perp}$ in the paramagnetic phase, using the hybridization-mediated interionic model Hamiltonian and a phenomenological single-ion CF term. Our results for the cerium monopnictides are discussed and compared with DCNS experiments in Sec. III A. The results for $\mathrm{PuSb}$ and the uranium 
monopnictides are presented and discussed in Secs. III B and III C, respectively. Finally, a brief summary of our results is given in Sec. IV.

\section{CALCULATION OF THE SUSCEPTIBILITY}

The hybridization between the quasilocalized $\mathrm{Ce}^{3+}\left(f^{1}\right)$ level and band electrons was first treated by Coqblin and Schrieffer ${ }^{11}$ using an Anderson-type model. ${ }^{32}$ Upon applying the Schrieffer-Wolff ${ }^{33}$ transformation they found in second-order perturbation theory an interionic interaction, mediated by band electrons, as in the case of the Ruderman-Kittel-Kasuya-Yosida interaction, ${ }^{34}$ but highly anisotropic. As has been shown by Siemann and Cooper, ${ }^{10}$ the predominant two-ion coupling in the $f^{1}$ case arises from the $m_{l}=0$ (quantization along the interionic axis) component of the $f$ wave function, corresponding to the piling up of charge along the interionic axis. This leads to a highly anisotropic interaction which acts to align the magnetic moments perpendicular to the interionic axis.

The resulting hybridization-mediated interaction Hamiltonian, in its Fourier-transformed form, can be writ$\operatorname{ten}^{25,35}$ as

$$
\mathscr{H}_{\mathrm{CS}}=-\sum_{\substack{\mathrm{q} \\ \sum_{\boldsymbol{\epsilon}, \boldsymbol{v}}}} J_{\mu \nu}^{\epsilon \sigma}(\mathbf{q}) L_{\mu \nu}^{\mathrm{q}} L_{\epsilon \sigma}^{-\mathrm{q}}
$$

where $\mu, v, \epsilon$, and $\sigma$ label the single-ion states of the $z$ component $M_{J}$ of the angular momentum $\left(J=\frac{5}{2}\right)$ along the [001] axis of quantization in the crystal; the $L_{\mu \nu}^{\mathrm{q}}$ are Fourier transforms of the standard-basis operators (transition operators), ${ }^{36} L_{\mu \nu}^{i} \equiv|\mu\rangle\langle\nu|$, which transfer the $\mathrm{Ce}^{3+}$ ion on site $i$ from state $|\nu\rangle$ to state $|\mu\rangle$; the anisotropic exchange coefficients $J_{\mu \nu}^{\epsilon \sigma}(\mathbf{q})$ are given by

$$
J_{\mu \nu}^{\epsilon \sigma}(\mathbf{q})=N^{-1} \sum_{\mathbf{R}_{i j}} E_{i j} e^{-i(\mu-v+\epsilon-\sigma) \phi_{i j}} B_{\mu \nu}^{\epsilon \sigma}\left(\theta_{i j}\right) e^{i \mathbf{q} \cdot \mathbf{R}_{i j}},
$$

where $\theta_{i j}$ and $\phi_{i j}$ are the angular coordinates of the interionic axis $\mathbf{R}_{i j}$ with respect to the axis of quantization, the function $B_{\mu \nu}^{\epsilon \sigma}$ is given in terms of the matrix elements of the $J$ th $\left(\frac{5}{2}\right.$ th $)$ irreducible representation of the full rotation group,,$^{37}$ and $E_{i j}$ are phenomenological interaction range parameters, with $E_{n}$ giving the interaction strength with the $n$th nearest neighbor.

To calculate the linear response of the system to a spatially varying external magnetic field, $H(\mathbf{r})=H \cos (\mathbf{q} \cdot \mathbf{r})$, applied parallel to the [001] direction (labeled as the $z$ axis), one adds to the Hamiltonian the Zeeman term

$$
\mathscr{H}_{\mathrm{ext}}=-\left(g \mu_{B} H / 2\right) \sum_{\mu, v} m_{\mu v}\left(L_{\mu \nu}^{\mathrm{q}}+L_{\mu v}^{-\mathrm{q}}\right),
$$

where $m_{\mu v}=\left\langle\mu\left|J^{z}\right| v\right\rangle$, and

$$
M_{z}^{\mathrm{q}} \equiv \sum_{\mu, v} M_{\mu \nu}^{\mathrm{q}}=g \mu_{B} \sum_{\mu, v} m_{\mu \nu} L_{\mu \nu}^{\mathrm{q}}
$$

denotes the Fourier transform of the $z$ component of the magnetic moment operator $M_{z}^{i}$, on site $i$, which as with any other single-ion operator can be expressed as a linear combination of the standard-basis operators. Note that since the single-ion basis states $|\mu\rangle$ are chosen so as to be the eigenstates of $\mathrm{J}^{z}, m_{\mu v}=\mu \delta_{\mu v}$. (For odd $f$ configurations as considered herein, $m_{\mu \mu}$ is nonvanishing always.) The two-ion interaction term is treated within the RPA, in which fluctuations of the Fourier components about their expectation values are neglected. Furthermore, for temperatures higher than that at which long-range order sets in, $\left\langle L_{\mu \nu}^{\mathrm{q}^{\prime}}\right\rangle$ may be replaced by $\left\langle L_{\mu \nu}^{\mathrm{q}^{\prime}}\right\rangle \delta_{\mathrm{qq}^{\prime}}$, because the only components with nonzero average values are those driven by the applied field. Thus, the effective RPA Hamiltonian in the paramagnetic regime becomes

$$
\begin{aligned}
\mathscr{H}_{\mathrm{RPA}}= & -2 \sum_{\mu, v} J_{\mu \nu}^{\epsilon \sigma}(\mathbf{q})\left(L_{\mu \nu}^{\mathrm{q}}\left\langle L_{\epsilon \sigma}^{-\mathrm{q}}\right\rangle+L_{\mu \nu}^{-\mathrm{q}}\left\langle L_{\epsilon \sigma}^{\mathrm{q}}\right\rangle\right) \\
& -\left(g \mu_{B} H / 2\right) \sum_{\mu, v} m_{\mu \nu}\left(L_{\mu \nu}^{\mathrm{q}}+L_{\mu \nu}^{-\mathrm{q}}\right),
\end{aligned}
$$

which defines the total effective field acting upon the qth component as

$H_{\mu \nu}(-\mathbf{q})_{\mathrm{eff}}=\frac{2}{g \mu_{B} m_{\mu \nu}} \sum_{\epsilon, \sigma} J_{\mu \nu}^{\epsilon \sigma}(\mathbf{q})\left\langle L_{\epsilon \sigma}^{-\mathrm{q}}\right\rangle+H / 2$.

From the definition of the longitudinal local susceptibility, $\chi_{i j}^{z z} \equiv d\left\langle M_{z}^{i}\right\rangle / d H_{z}^{j}$ (where for simplicity of notation the $z$ superscripts will be omitted), the expectation value of the Fourier transform components of $M_{z}^{\mathrm{q}}$ can be expressed either in terms of the applied field $H(\mathbf{q})=H / 2$, or in terms of the effective field, in the form

$$
\left\langle M_{\mu \nu}(\mathbf{q})\right\rangle=\chi_{\mu \nu}(\mathbf{q}) H / 2=\chi_{\mu \nu}^{(0)}(\mathbf{q}) H_{\mu \nu}(\mathbf{q})_{\mathrm{eff}},
$$

where

$$
\chi_{\mu \nu}^{(0)}(\mathbf{q})=N\left(g \mu_{B}\right)^{2} \frac{\mu^{2}}{(2 J+1) k_{B} T} \delta_{\mu \nu}
$$

is the single-ion (noninteracting) component of the susceptibility at high temperatures, satisfying the sum rule $\chi^{(0)}(\mathbf{q})=\sum_{\mu} \chi_{\mu \mu}^{(0)}(\mathbf{q})$. Inserting Eq. (6) in Eq. (7) and solving for $\chi_{\mu \mu}(\mathbf{q})$ we find

$$
\chi_{\mu \mu}(\mathbf{q})=\chi_{\mu \mu}^{(0)}(\mathbf{q})+2 \chi_{\mu \mu}^{(0)}(\mathbf{q}) \sum_{\epsilon} \widetilde{J}_{\mu \mu}^{\epsilon \epsilon}(\mathbf{q}) \chi_{\epsilon \epsilon}(\mathbf{q}),
$$

where

$$
\widetilde{J}_{\mu \mu}^{\epsilon \epsilon}(\mathbf{q})=J_{\mu \mu}^{\epsilon \epsilon}(\mathbf{q}) /\left(g^{2} \mu_{B}^{2} m_{\mu \mu} m_{\epsilon \epsilon}\right) .
$$

Upon solving the set of $6 \times 6$ linear equations for $\chi_{\mu \mu}(q)$, we find that the qth component of the longitudinal susceptibility in the paramagnetic phase is given by

$$
\begin{aligned}
\chi(\mathbf{q}) & =\sum_{\mu} \chi_{\mu \mu}(\mathbf{q}) \\
& =\sum_{\mu, \epsilon}\left\{\left[\underline{\mathbb{1}}-2 \underline{\chi}^{(0)}(\mathbf{q}) \underline{\widetilde{J}}(\mathbf{q})\right]^{-1}\right\}_{\mu \epsilon} \chi_{\epsilon \epsilon}^{(0)}(\mathbf{q}),
\end{aligned}
$$

where an underline denotes a $6 \times 6$ matrix in the free-ion basis-state representation. Although this result is similar to the familiar expression of the susceptibility for the Heisenberg exchange Hamiltonian, it is more complicated in form due to the anisotropic nature of the exchange coefficients. Note that even though the above formalism was derived for the simple $f^{1}$ case, it is exactly identical for the general case of $f^{n}$ systems, except for the angular dependence of the matrix elements $B_{\mu \nu}^{\epsilon \sigma}(\theta)$, in Eq. (2), 
which varies with the number $(n)$ of $f$ electrons in the ion and the nature of intraionic coupling. As $T$ decreases from infinity, a second-order phase transition occurs at some temperature $T_{N}$, when the determinant of the matrix $\underline{1}-2 \underline{\chi}^{(0)}\left(q_{0}\right) \underline{\widetilde{J}}\left(q_{0}\right)$ vanishes for some wave vector $q_{0}$ which determines the periodicity of the magnetically ordered structure below $T_{N}$. $\left[q_{0}=(2 \pi / a)(0,0,1)\right.$ for the AF-I structure.]

In deriving Eq. (11) we have assumed that the crystalfield interaction is smaller than the hybridizationmediated two-ion coupling and have thus neglected the $\mathrm{CF}$ effects. However, on going to the lighter compounds one must take into account both of these effects on an equal footing. Thus, in addition to the two-ion interaction of Eq. (1) we include a cubic CF Hamiltonian of the form $^{38}$

$$
\mathscr{H}_{\mathrm{CF}}=B_{4} \sum_{i}\left[O_{4}^{0}(i)+5 O_{4}^{4}(i)\right],
$$

where the $O_{n}^{m}$ are the Stevens ${ }^{39}$ operator equivalents and the CF interaction parameter $B_{4}$ is treated phenomenologically. For the $f^{1}$ and $f^{5}$ systems $\left(J=\frac{5}{2}\right)$, the CF splitting $\Delta_{\mathrm{CF}}$, between the $\Gamma_{7}$ doublet (ground state for positive $B_{4}$ ) and the $\Gamma_{8}$ quartet is given by $\Delta_{\mathrm{CF}}=360 B_{4}$. For the $f^{3}$ systems, the CF interaction acting on the $J=\frac{9}{2}$ manifold, which in addition to the fourth-order term given by Eq. (12) also contains a sixth-order term, leads to two quartets $\Gamma_{8}^{(1)}, \Gamma_{8}^{(2)}$ and a Kramers doublet $\Gamma_{6}$. The crystal field is often specified in terms of another equivalent set of parameters, $W$ and $x$ (in the notation of Ref. 38), with the overall CF splitting being directly proportional to the strength $W$. In the presence of the CF Hamiltonian term it can be shown that a RPA treatment in the paramagnetic phase yields an expression for the longitudinal component of the susceptibility which is identical in form to Eq. (11), but with the exchange coefficients $\widetilde{J}_{\mu \mu}^{\epsilon \epsilon}(\mathbf{q})$, containing an additional $\mathbf{q}$-independent term proportional to the CF parameter $B_{4}$.

The correlation lengths of the individual fluctuations near the ordering temperature can be calculated within the Ornstein-Zernike ${ }^{40}$ approximation by expanding $\chi^{Z z}(q)$ about $q_{0}$, or equivalently from ${ }^{41}$

$$
\xi_{\alpha}^{2}=\kappa_{\alpha}^{-2}=-\frac{1}{2}\left[g^{z z}(\mathbf{q})\right]^{-1} \frac{\partial^{2} g^{z z}(\mathbf{q})}{\partial \mathbf{q}_{\alpha}^{2}},
$$

evaluated at $\mathrm{q}_{0}$, where $\alpha=x, y, z$ and

$$
g^{z z}(\mathbf{q})=k_{B} T \chi^{z z}(\mathbf{q})
$$

is the Fourier transform of the correlation function.

\section{RESULTS AND COMPARISON WITH EXPERIMENT}

\section{A. Cerium systems}

The anisotropic behavior of the longitudinal fluctuations of the magnetization or of the q-dependent susceptibility in Eq. (11) follows in a natural way from the anisotropy of the hybridization-mediated exchange coefficients $J_{\mu \mu}^{\epsilon \epsilon}(q)$. The majority of these matrix elements show ${ }^{24,25}$ a flat dispersion in the [001] direction and marked disper- sion in other directions, resulting in long-range correlations within (001) planes and short-range correlations between (001) planes. (For the simpler case of an isotropic Heisenberg Hamiltonian, $\xi^{2} \sim\left|d^{2} J(\mathbf{q}) / d q^{2}\right|_{\mathbf{q}=\mathbf{q}_{0}}$.) Furthermore, it is important to note that in contrast to the conventional susceptibility expression for the isotropic Heisenberg Hamiltonian, upon taking the matrix inversion, $\chi(q)$ in Eq. (11) may exhibit several poles corresponding to different types of magnetic ordering, which can lead to a multicritical behavior.

The previous calculations for the equilibrium ${ }^{24}$ and excitation $^{25}$ magnetic behavior of the heavier monopnictides, performed with nearest-neighbor $\left(E_{1}\right)$ and secondnearest-neighbor $\left(E_{2}\right)$ ferromagnetic two-ion coupling constants, have shown that for a fairly wide range of values of $E_{2} / E_{1}$ near $E_{2}=E_{1}$ the lattice geometry strongly favors a ferromagnetic [001] moment alignment. Figure 1 shows the variation of the inverse critical correlation lengths (multiplied by the lattice constant $a$ ) parallel and perpendicular to the [001] moment direction, at the reduced temperature $\left(T-T_{c}\right) / T_{c}=0.04$, as a function of the ratio $E_{2} / E_{1}$ for ferromagnetic ordering $\left(q_{0}=0\right)$. As one can see from Fig. 1, $\kappa_{\perp}$ is remarkably independent of the range of the CS exchange interactions and is smaller than $\kappa_{\|}$for a rather broad range of $E_{2} / E_{1}$ values. This result clearly shows the strong and longrange nature of the intraplanar interactions resulting from the highly anisotropic angular dependence of the exchange coefficients. (Distortional effects associated with ferromagnetism are not expected to modify Fig. 1 to any significant extent.) On the other hand, the range of the interplanar correlations strongly depends on the range of the hybridization-mediated anisotropic exchange coupling constants. As $E_{1}$ predominates (exchange interaction becomes "short range") $\xi_{\|}$is smaller relative to $\xi_{\perp}$ and increases rapidly in size as the anisotropic exchange be-

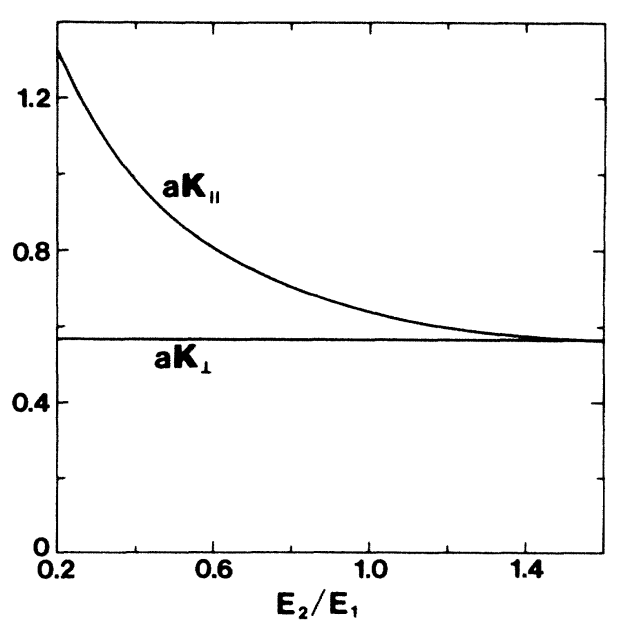

FIG. 1. Inverse critical correlation lengths for the longitudinal fluctuations parallel $\left(\kappa_{||}\right)$and perpendicular $\left(\kappa_{\perp}\right)$ to the [001] moment direction for ferromagnetic ordering vs ratio of nextnearest $\left(E_{2}\right)$ to nearest-neighbor $\left(E_{1}\right)$ anisotropic hybridization-mediated (CS-type) coupling, for systems of $\mathrm{Ce}^{3+}$ on an fcc lattice with lattice constant $a$. 
comes further "long range," approaching asymptotically the value of $\xi_{\perp}$. The corresponding variation of $\kappa_{\|} / \kappa_{1}$ with the ratio $E_{2} / E_{1}$ near the ordering temperature $\left(T-T_{c}=0.04 T_{c}\right.$ ) for the ferromagnetic case (F) is shown in Fig. 2.

The addition of a small antiferromagnetic nearestneighbor (NN) isotropic exchange interaction ${ }^{25} H_{1}$, or a third NN anisotropic CS-type interaction ${ }^{26} E_{3}$, to the anisotropic ferromagnetic interactions $E_{1}$ and $E_{2}$ was shown to stabilize the type-I AF ground state. For the $E_{1}=E_{2}=\left|E_{1}\right|$ case, even though the AF-I phase is stabilized for relatively small $H_{1}=0.0217 E_{1}$ or $E_{3}$ $=-0.1 E_{1}$, a rather large $H_{1}=-0.4 E_{1}$ or $E_{3}=-0.3 E_{1}$ is required in order to reproduce the experimental intensity distribution of the excitation spectrum ${ }^{25,26}$ in $\mathrm{CeBi}$. Our numerical results for $\kappa_{\|} / \kappa_{\perp}$, evaluated in the vicinity of the Néel temperature $\left(T-T_{N}=0.04 T_{N}\right)$, are displayed in Fig. 2 as a function of $E_{2} / E_{1}$ for various values of $E_{3} / E_{1}$, which yield an AF-I ordering. As one can see from Fig. 2, upon making the transition to the type-I antiferromagnet, there is a major quantitative enhancement (by a factor of about 1.7) in the size of $\kappa_{||} / \kappa_{\perp}$ for a wide range of $E_{2} / E_{1}$. We find that the addition of a small antiferromagnetic $E_{3}$ CS-type (hybridization-mediated) interaction has almost no effect on $\kappa_{\perp}$ whereas it increases $\kappa_{\|}$, thus making the correlations along the moment direction further short range. This effect is a direct result of the relative flatness ${ }^{24,25}$ of the dispersion curves of the exchange coefficients $J_{\mu \mu}^{\epsilon \epsilon}(\mathbf{q})$, near $\mathrm{q}_{0}=(2 \pi / a)(0,0,1)$ compared to the behavior near $q_{0}=0$. The discontinuity in $\kappa_{\|} / \kappa_{1}$ occurring at $E_{2} / E_{1}=1.3$ for $E_{3} / E_{1}=-0.1$ arises from the preference of the system to order in a ferromagnetic rather than in an AF-I structure, as $E_{2} / E_{1}$ increases. Note that for $E_{3}=-0.3 E_{1}$ and $E_{2}=E_{1}=\left|E_{1}\right|$

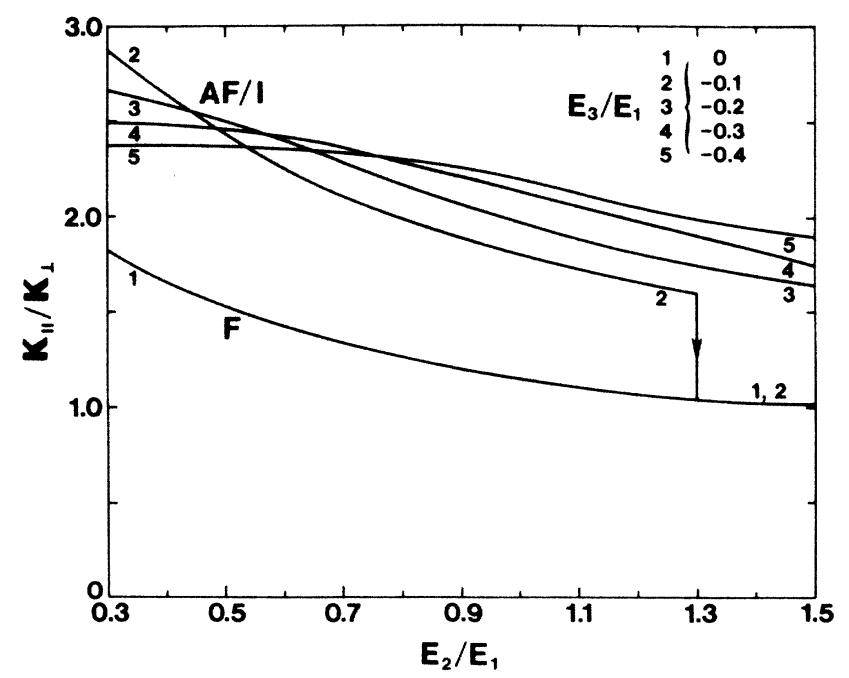

FIG. 2. Variation of ratio of inverse critical correlation lengths parallel and perpendicular to the [001] moment direction with the ratio of next-nearest $\left(E_{2}\right)$ to nearest-neighbor $\left(E_{1}\right)$ anisotropic CS-type coupling, for ferromagnetic (F) ordering $\left(E_{3} / E_{1}=0\right)$ and four cases of type-I antiferromagnetic ordering (AF-I), respectively. The results are for systems of $\mathrm{Ce}^{3+}$ on an fcc lattice. (parameter values previously used $^{26}$ for the excitation spectrum of $\mathrm{CeBi}$ ) and in the absence of a CF interaction, we find that $\kappa_{\|} / \kappa_{1}=2.15$, in good agreement with the experimental value ${ }^{13}$ of $2.5 \pm 0.2$ for $\mathrm{CeBi}$. On the other hand, performing the calculation with an isotropic $H_{1}$ instead of an anisotropic $E_{3}\left(H_{1}=-0.1 E_{1}\right.$ and $\left.E_{2}=E_{1}\right)$, we find the rather small value of 1.5 for $\kappa_{\|} / \kappa_{\perp}$, indicating that an AF third NN anisotropic exchange interaction enhances the intra- to interplanar anisotropy.

Figure 3 shows the variation of inverse correlation lengths (multiplied by the lattice constant $a$ ) parallel and perpendicular to the moment direction with reduced temperature above $T_{N}$ for the case of AF-I ordering with $E_{1}=E_{2}=\left|E_{1}\right|$ and $E_{3}=-0.3 E_{1}$. Also shown in Fig. 3 is the ratio $\kappa_{\|} / \kappa_{1}$, which is approximately independent of temperature, as is experimentally observed. Furthermore, we find values for the critical exponents $v_{\|}$and $v_{\perp}$ associated with $\kappa_{\|}$and $\kappa_{\perp}\left[\kappa \cong\left(T-T_{N}\right)^{v}\right]$ of $0.56 \pm 0.05$ and $0.53 \pm 0.05$, respectively, compared to the experimental value $^{13}$ of $0.63 \pm 0.06$ for $\mathrm{CeBi}$. The departure of our calculated values of $v$ from the mean-field value of 0.5 comes from the approximate numerical procedures used (essentially evaluating $v$ from the average behavior in the temperature range $\left.0.004 \leq\left(T-T_{N}\right) / T_{N} \leq 0.10\right)$.

The effect of including the cubic CF interaction is shown in Fig. 4, where the ratio $\kappa_{\|} / \kappa_{1}$, evaluated at $T-T_{N}=0.04 T_{N}$, is plotted as a function of the ratio of CF splitting $\Delta_{\mathrm{CF}}$, to the nearest-neighbor anisotropic exchange interaction $E_{1}$ for AF-I ordering with $E_{2}=E_{1}$ and various values of $E_{3} / E_{1}$. Upon increasing $\Delta_{\mathrm{CF}} / E_{1}$ ( $\Gamma_{7}$ ground state), the ratio $\kappa_{\|} / \kappa_{\perp}$ gradually decreases and for sufficiently large CF splitting $\kappa_{\|} / \kappa_{\perp}$ becomes smaller than unity, indicating the reversal of the relative strength of intra- to interplanar coupling. The reduction of $\kappa_{\|} / \kappa_{\perp}$

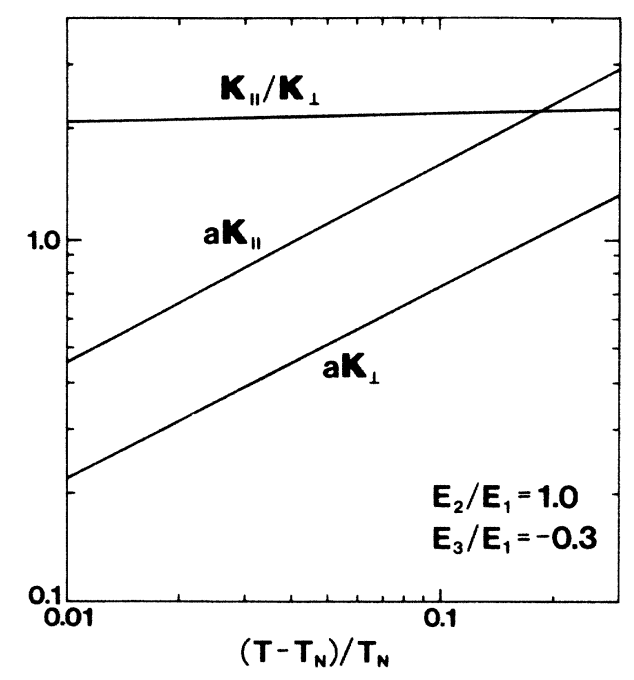

FIG. 3. Inverse correlation lengths for the longitudinal fluctuations parallel $\kappa_{\|}$and perpendicular $\kappa_{\perp}$ to the [001] moment direction as a function of reduced temperature above $T_{N}$ for systems of $\mathrm{Ce}^{3+}$ ions on an fcc lattice with lattice constant $a$. The magnetic ordering at $T_{N}$ is antiferromagnetic of type-I (AF-I). Also shown is the temperature variation of $\kappa_{\|} / \kappa_{\perp}$. 


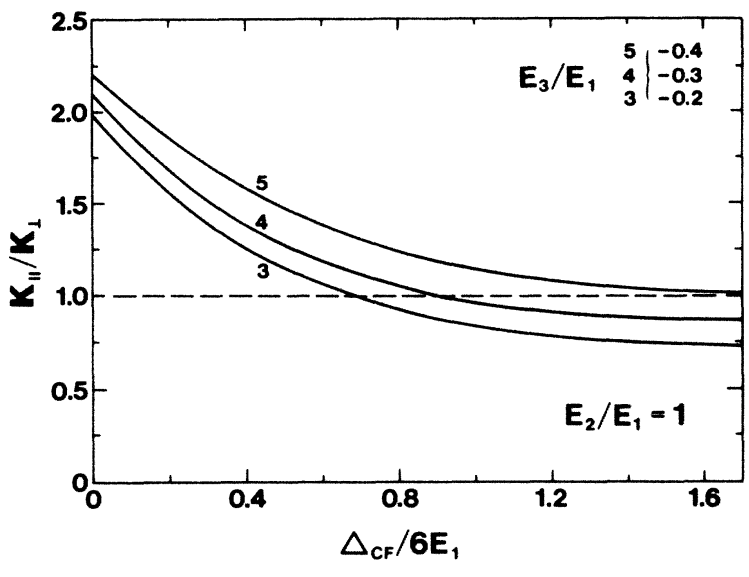

FIG. 4. Ratio of inverse critical correlation lengths for the longitudinal fluctuations parallel and perpendicular to the [001] moment direction as a function of the ratio of the crystal-field splitting to the nearest-neighbor CS-type anisotropic coupling, for $\mathrm{Ce}^{3+}\left(f^{1}\right)$ compounds having type-I antiferromagnetic ordering at $T_{N}$ for $E_{2}=E_{1}$.

with increased crystal-field strength arises from the competition between two-ion and crystal-field anisotropy. The two-ion interaction favors an $\langle 001\rangle$ easy axis, whereas a crystal field of a sign corresponding to effective negative charges associated with nearest-neighbor anion sites favors a $\langle 111\rangle$ easy axis. ${ }^{9}$ Thus increasing $\Delta_{\mathrm{CF}}$ compared to $E_{1}$ greatly reduces the range of correlations within the (001) planes, and consequently decreases the ratio $\kappa_{\|} / \kappa_{1}$. The crossover (in reciprocal space) from the cigarlike shape $\left(\kappa_{\|}>\kappa_{\perp}\right)$ to the lenslike shape $\left(\kappa_{\|}<\kappa_{\perp}\right)$ occurs at $\Delta_{\mathrm{CF}}=6.6 T_{N}\left(T_{N}=0.65 E_{1}\right)$ for $E_{3}=-0.2 E_{1}$ and at $\Delta_{\mathrm{CF}}=10.8 T_{N}\left(T_{N}=0.57 E_{1}\right)$ for $E_{3}=-0.3 E_{1}$.

Figure 5 displays the calculated values of $\kappa_{\|} / \kappa_{\perp}$, along with those from diffuse critical neutron scattering experi-

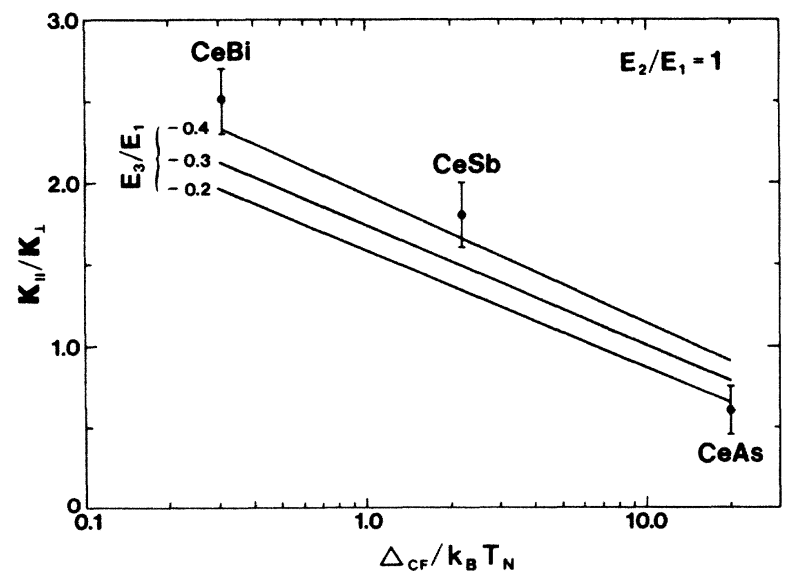

FIG. 5. Calculated ratio of inverse critical correlation lengths parallel and perpendicular to the [001] moment direction vs ratio of the crystal-field splitting to the ordering temperature for $\mathrm{Ce}^{3+}\left(f^{1}\right)$ compounds having type-I antiferromagnetic ordering with $E_{2}=E_{1}$. The closed circles are the experimental results from diffuse critical neutron scattering experiments (Refs. 5, 12, 13 , and 14). ments on Ce monopnictides, ${ }^{12}$ as a function of the ratio of the CF splitting to the Neel temperature with $E_{2}=E_{1}$ and various values of $E_{3} / E_{1}$. Our numerical results (in particular, those obtained with $E_{3}=-0.3 E_{1}$ ) are in good agreement with experiment, further substantiating the experimental relevance of the hybridization-mediated interaction.

\section{B. Plutonium systems}

The initial calculations for the equilibrium magnetic behavior of $\mathrm{Pu}^{3+}\left(f^{5}\right)$ systems were performed in the $j-j$ and the $L-S$ intraionic coupling limits. ${ }^{4,28}$ The angular dependence of the interaction in the $j-j$ limit has been shown ${ }^{28}$ to be the same for the $f^{n}$ and $f^{6-n}$ ions, i.e., the same for $\mathrm{Pu}^{3+}\left(f^{5}\right)$ as for $\mathrm{Ce}^{3+}\left(f^{1}\right)$. While the magnitude of the moment and the behavior of the magnetization with temperature were well reproduced in the $L-S$ limit, no phase transition from a ferromagnetic to an AF-I phase could be obtained, i.e., behavior qualitatively modeling the ferromagnetic to antiferromagnetic transition which occurs ${ }^{3,4}$ in PuSb. On the other hand, in the limit of $j-j$ coupling, a transition to AF-I behavior was obtained, but the size of the moment and the variation of the magnetization with temperature in the ferromagnetic phase could not be reproduced. Subsequently, Banerjea et $a l .{ }^{29}$ extended the theory to the case of intermediate coupling, including the possibility of long-period AF structures. The theory then reproduced the experimentally observed magnetic behavior of PuSb, predicting a phase transition from a low-temperature $F$ phase to a longperiod AF phase at about $75 \mathrm{~K}$, with ordered moments close to the experimental values. However, while the moment polarization in the long-period AF phase has been experimentally observed to be longitudinal with respect to the direction of modulation, the theory predicted a transverse polarization with moments aligned along the cube edge.

The variation of $\kappa_{\|} / \kappa_{\perp}$, evaluated in the vicinity of the ordering temperature $\left(T-T_{c}=0.04 T_{c}\right)$, is shown in Fig. 6 as a function of $E_{2} / E_{1}$, for ferromagnetic ordering $\left(\mathrm{q}_{0}=0\right)$ and for the $j-j, L-S$, and intermediate coupling cases. As one can see from Fig. 6, our numerical results for $\kappa_{||} / \kappa_{\perp}$ in the IC case and the $L-S$ case are very similar. This is not surprising since the ground state of the $L-S$ coupling limit is the predominant component of the IC ground state, which we take ${ }^{29}$ as $75 \%{ }^{6} H$ and $25 \%{ }^{4} G$. More importantly, in both the $L-S$ and IC cases $\kappa_{\|} / \kappa_{\perp}$ is smaller than unity (lenslike shape in reciprocal space) and is almost independent of the range of the exchange interactions. On the other hand, on going to the $j-j$ coupling limit (ceriumlike behavior) there is a quantitative change in the size $\left(\kappa_{\|} / \kappa_{1}>1\right)$ and variation of $\kappa_{\| \mid} / \kappa_{1}$ with $E_{2} / E_{1}$ from that found in the $L-S$ coupling limit. This markedly different behavior of $\kappa_{\|} / \kappa_{\perp}$ in the $j-j$ and $L-S$ coupling limits arises from the different angular dependence of the coefficients $B_{\mu \nu}^{\epsilon \sigma}(\theta)$, in these two limits (see Fig. 1 of Thayamballi and Cooper ${ }^{28}$ ).

The addition of a small AF $H_{3}\left(-0.028 E_{1}\right.$ for IC) or a moderate AF $E_{3}\left(-0.32 E_{1}\right.$ for IC and $-0.33 E_{1}$ for $L-S$ coupling) has been found ${ }^{29}$ to produce a transition to a 3,3 


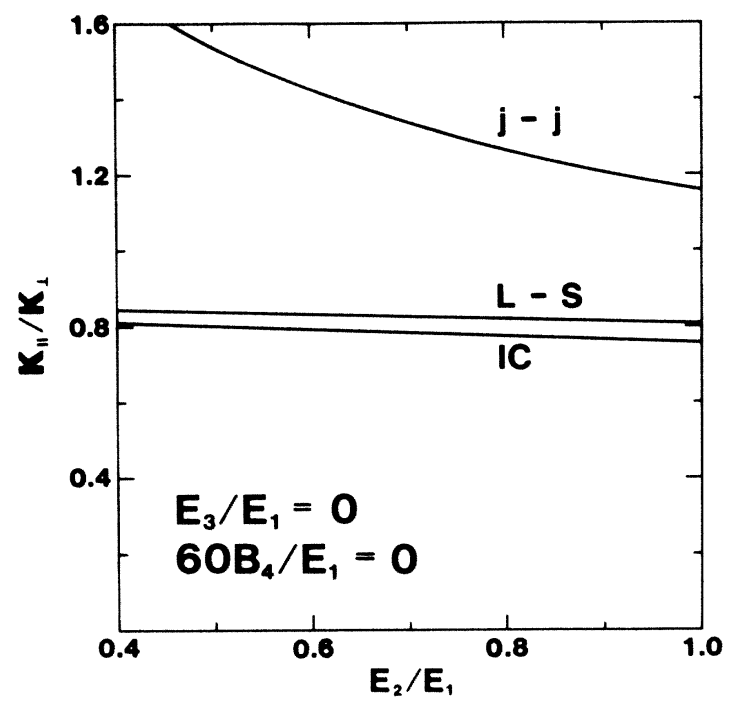

FIG. 6. Ratio of inverse critical correlation lengths parallel and perpendicular to the [001] moment direction for $\mathrm{Pu}^{3+}\left(f^{5}\right)$ fcc systems as a function of $E_{2} / E_{1}$, for ferromagnetic ordering $\left(E_{3} / E_{1}=0\right)$ and three cases of intraionic coupling. The IC ground state is taken as composed of $75 \%{ }^{6} \mathrm{H}$ and $25 \%{ }^{4} \mathrm{G}$, as in Ref. 29.

structure, in which one has ferromagnetic planes in a three-up-three-down arrangement with a moment along the cube edge but perpendicular to the modulation direction. Moreover, a large negative $\left(B_{4}<0\right)$ CF splitting was included $^{29}$ (yielding $\Gamma_{8}$ as the CF ground state) in order to reproduce the experimentally observed low-temperature moment ${ }^{42}$ of $0.76 \mu_{B}$ in PuSb. Using the same values for $H_{3}$ or $E_{3}$ and varying $\Delta_{\mathrm{CF}}$ over a wide range of negative values, we find that in both IC and $L-S$ coupling cases, $\kappa_{\|}$ becomes imaginary near the ordering temperature for AF-I or AF-IA $(++--$ sequence) ordering. This result, which is consistent with those of the equilibrium magnetic structure calculations, ${ }^{29}$ suggests that it is not possible to reproduce a transition from the paramagnetic state to a type-I or -IA antiferromagnetic structure with physically reasonable values for the correlation lengths (i.e., the long-period structure is essential to having an antiferromagnetic transition).

The variation of $\kappa_{\|} / \kappa_{1}$, evaluated at $T-T_{N}=0.04 T_{N}$, with negative CF splitting ( $\Gamma_{8}$ low) is shown in Fig. 7 for IC and $L-S$ coupling for the case of transversely polarized long-period AF structures (3,3 and 5,5). Note that the moment direction is taken to be along the [001] direction. Also displayed in Fig. 7 for comparison are the numerical results for $\kappa_{||} / \kappa_{1}$ in the $j-j$ coupling limit for the longitudinally polarized AF-I structure. (All results in Fig. 7 are for $E_{2}=E_{1}=\left|E_{2}\right|$ and $E_{3}=-0.32 E_{1}$.) However, while a longitudinal AF-I structure occurs in the $j-j$ case, we have not been able to reproduce a transition to a longitudinally polarized long-period AF structure in the $j-j$ coupling scheme with real (physical) values for the correlation lengths. As in Fig. 6, our numerical results for $\kappa_{\|} / \kappa_{\perp}$ in the IC case are very similar to those in the $L-S$ coupling case as one would expect. It is interesting to note in Fig. 7, that the difference between the IC and the $L-S$

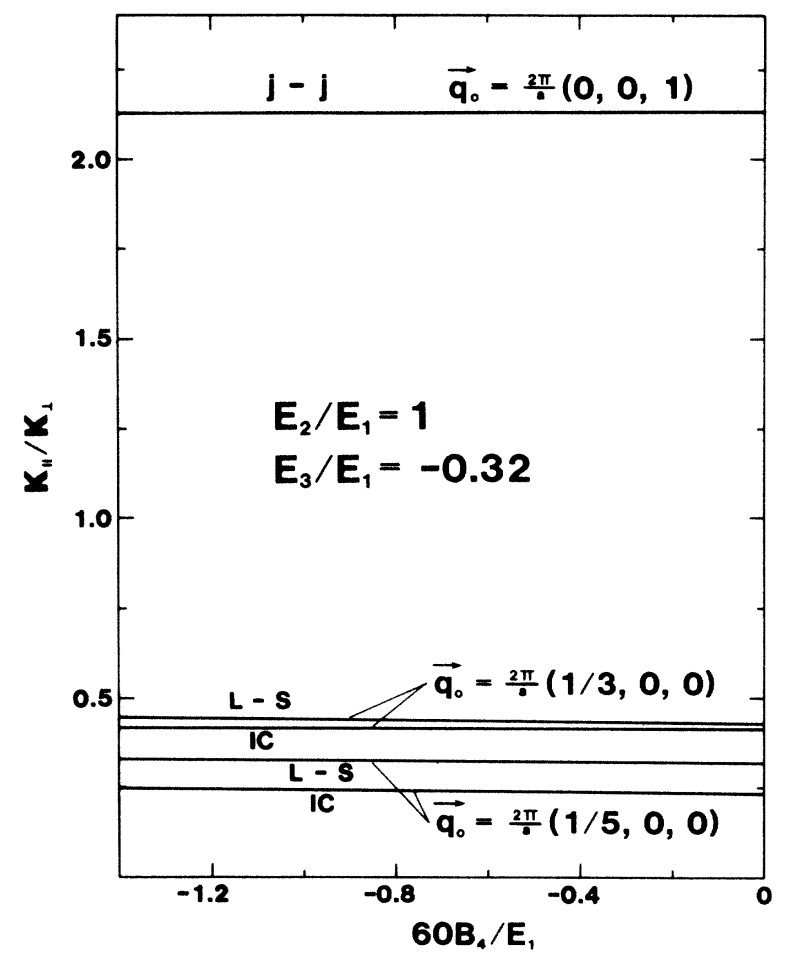

FIG. 7. Ratio of inverse critical correlation lengths parallel and perpendicular to the [001] moment direction for $\mathrm{Pu}^{3+}\left(f^{5}\right)$ systems, with $E_{2}=E_{1}$ and $E_{3}=-0.32 E_{1}$, plotted as a function of the ratio of the crystal-field parameter ${ }^{38} B_{4}$ to the nearestneighbor exchange coupling for three cases of intraionic coupling and various types of AF structures, labeled with the appropriate modulation vector $\mathbf{q}_{0}$. The IC ground state is taken as composed of $75 \%{ }^{6} \mathrm{H}$ and $25 \%{ }^{4} \mathrm{G}$, as in Ref. 29.

curves decreases sharply on going from the $\mathbf{q}_{0}$ $=(2 \pi / a)\left(\frac{1}{5}, 0,0\right)$ to the $\mathrm{q}_{0}=(2 \pi / a)\left(\frac{1}{3}, 0,0\right)$ antiferromagnet. Thus if the $(2 \pi / a)(1,0,0)$ antiferromagnet provided a stable solution (which, as already stated, it does not), it would appear that the IC curve might fall slightly above the $L-S$ curve.

As one can see from Fig. 7, in all three coupling cases $\kappa_{\|} / \kappa_{\perp}$ is essentially independent of the CF splitting between the $\Gamma_{8}$ ground state and the $\Gamma_{7}$ doublet. This saturation of the anisotropy in correlation lengths is a consequence of the fact that a $\Gamma_{8}$ ground state favors moment alignment along the $\langle 001\rangle$ direction. On the other hand, the numerical results for $\kappa_{\|} / \kappa_{\perp}$ in Fig. 4 show, as discussed above, that a CF of a sign having the $\Gamma_{7}$ doublet below the $\Gamma_{8}$ quartet (favoring $\langle 111\rangle$ easy direction at saturation $^{9}$ ) greatly changes the anisotropy in correlation lengths. More importantly, as can be seen in Fig. 7, there is a fundamental difference in the direction of the anisotropy in correlation lengths on going from $j-j$ to $L-S$ (or IC) intraionic coupling. The change of the critical scattering from cigarlike shape $\left(\kappa_{\|}>\kappa_{\perp}\right)$ in reciprocal space in the $j-j$ coupling case to lenslike shape $\left(\kappa_{\|}<\kappa_{\perp}\right)$ in the $L-S$ (or IC) coupling case arises from the different angular dependence of the two-ion interaction for $\mathrm{Pu}^{3+}$ for these differing intraionic couplings. ${ }^{28}$ This angular dependence of $B_{\mu \nu}^{\epsilon \sigma}(\theta)$ near the $L-S$ limit is also the mech- 
anism responsible for the transverse polarization of the $\mathrm{Pu}^{3+}\left(f^{5}\right)$ system. In particular, the strong anisotropy of $B_{5 / 2}^{5 / 25 / 2}$ and $B_{5 / 2}^{-5 / 2} 5 / 2{ }_{5 / 2}^{-5 / 2}$ (Fig. 1 of Ref. 28) with peaks at $\theta=0$ leads to a preference for moment alignment in planes transverse to the modulation direction of the structure, and corresponds to a strong piling up of charge along the [001] direction and hence strong interplanar rather than intraplanar interactions.

\section{Uranium systems}

The calculations for the equilibrium magnetic behavior of the $\mathrm{U}^{3+}\left(f^{3}\right)$ compounds, which were initially performed in the $j-j$ intraionic coupling limit, ${ }^{27}$ have been recently extended to the more realistic $L-S$ coupled case. ${ }^{30}$ These calculations illustrate the occurrence of the collinear to noncollinear thermally induced magnetic structural transition and also the stabilization of the triple- $k$ type-I structure. Furthermore, the results indicate that while systems with nearest-neighbor interactions alone are poor in noncollinear structures, systems with longer-range hybridization-mediated (CS) interaction do yield more noncollinear structures, with transitions to collinear structures as the temperature increases. Here we investigate the effect of varying interionic coupling (varying $\left.E_{2} / E_{1}, E_{3} / E_{1}\right)$ on the size of $\kappa_{\|} / \kappa_{1}$ near the ordering temperature for the case of $f^{3}$ systems with either $L-S$ or $j$-j intraionic coupling. $\left[\mathrm{U}^{3+}\left(f^{3}\right)\right.$ while having intermediate coupling, is close to the $L-S$ limit.] We relate the behavior to that of UAs, UP, and USb.

The angular variation of the fully diagonal $B$ 's for saturated ionic moment in $\mathrm{U}^{3+}\left(f^{3}\right)$ compounds is shown in Fig. 8, for the $j-j$ and $L-S$ intraionic coupled cases. The

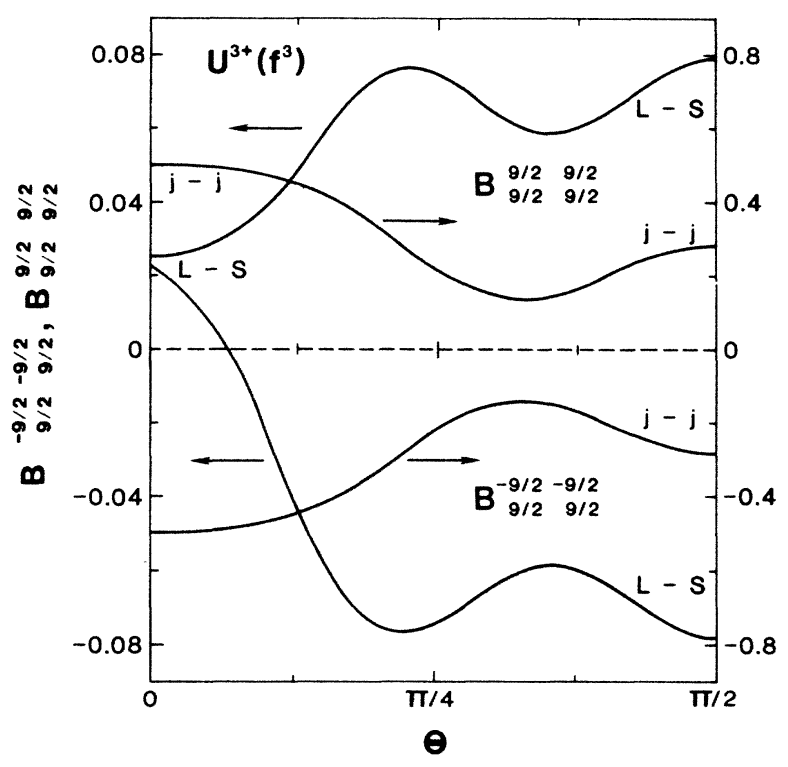

FIG. 8. Angular dependence of the two-ion diagonal matrix elements for coupling between $\mathrm{U}^{3+}$ ions with saturated parallel and antiparallel moments, for the $j-j$ and $L-S$ intraionic coupling limits. The upper indices label the $M_{j}$ states on one ion and the lower indices label the states of the other ion; $\theta$ is the angle between the interionic axis and the axis of moment alignment. matrix elements $B_{9 / 2}^{9 / 2} 9 / 2(\theta)$ and $B_{9 / 2}^{-9 / 2} 9 / 2-9 / 2(\theta)$ characterize the anisotropy of coupling for a pair of $\mathrm{U}^{3+}$ ions, when both have saturated moments that are parallel and antiparallel, respectively. (Here $\theta$ is the angle between the interionic axis and the [001] quantization axis.) The peak at $\theta=\pi / 2$ and the very low value at $\theta=0$ for the $L-S$ coupling limit, correspond to strong coupling within $\{001\}$ planes and weak coupling between planes. It is important to note that the corresponding angular behavior for the $L-S$ coupled $\mathrm{Pu}^{3+}\left(f^{5}\right)$ compounds behaves oppositely, with the anisotropy peak occurring at $\theta=0$ rather than at $\theta=\pi / 2$.

Figure 9 shows the variation of $\kappa_{\|} / \kappa_{1}$, evaluated at $T-T_{N}=0.04 T_{N}$, for $\mathrm{U}^{3+}\left(f^{3}\right)$ compounds, as a function of $E_{2} / E_{1}$ for the $j-j$ and $L-S$ coupling cases and for various values of $E_{3} / E_{1}$. In all cases the magnetic structure below the ordering temperature is AF of type I. The cigarlike shape $\left(\kappa_{\|}>\kappa_{1}\right)$ of the critical scattering for $f^{3}$ systems, in both the $j-j$ and $L-S$ coupling limits and for a wide range of the anisotropic exchange coupling parameters, results from the angular variation of the exchange matrix elements, such as those shown in Fig. 8 for the saturated moment states. We note that as the range of the anisotropic interionic exchange becomes long range $\left(E_{2} \sim E_{1}\right), \kappa_{||} / \kappa_{\perp}$ is almost independent of the type of intraionic correlations $(L-S$ or $j-j)$. On the other hand, as $E_{1}$ predominates and the exchange becomes short range, the type of intraionic coupling has a quantitative effect on the size of $\kappa_{\|} / \kappa_{\perp}$. This reflects the situation that what matters in determining the magnetic structure in the ordered state, and the associated anisotropy in critical behavior, is not just the gross shape of exchange matrix

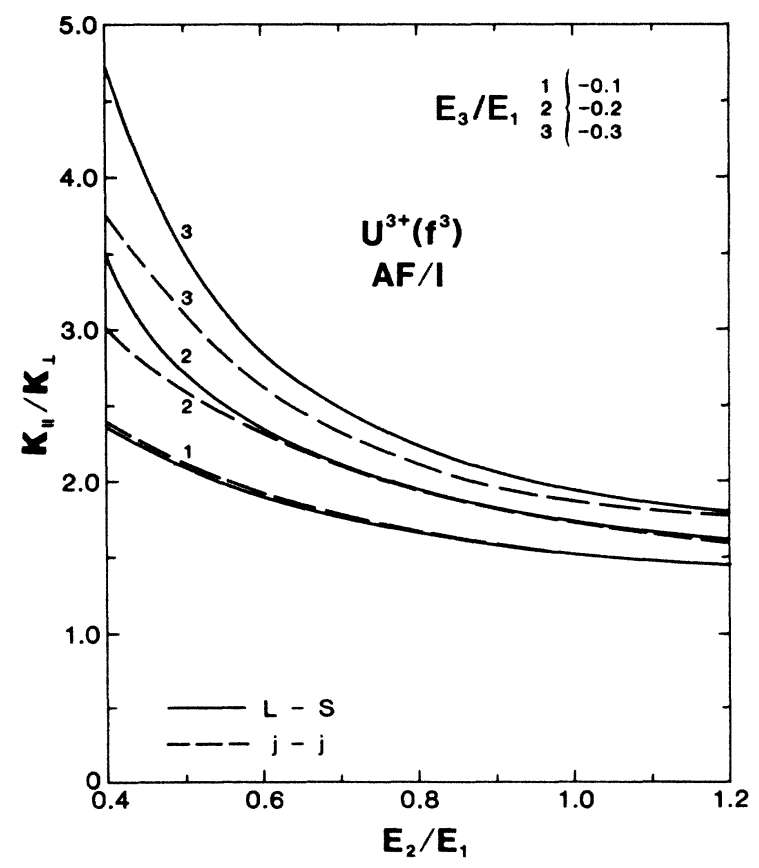

FIG. 9. Ratio of inverse critical correlation lengths parallel and perpendicular to the [001] moment direction for $\mathrm{U}^{3+}\left(f^{3}\right)$ fcc systems as a function of $E_{2} / E_{1}$, for $L-S$ and $j-j$ intraionic coupling and various values of $E_{3} / E_{1}$. In all cases the magnetic ordering at $T_{N}$ is antiferromagnetic of type-I (AF-I). 
element curves such as those shown in Fig. 8, but rather in detail where the angular orientation of the various neighbors falls along such curves and the difference in matrix element values between the parallel and antiparallel moment curves at such angular orientations. This was pointed out in the original discussion by Siemann and Cooper. $^{10}$

We next present the results of the calculations for $\mathrm{U}^{3+}\left(f^{3}\right)$ systems including the effect of CF splitting. Experimental estimates of the CF parameters are ${ }^{22}$ that $V_{4} \sim 3200 \mathrm{~K}$ and $V_{6} \sim 30 \mathrm{~K}$ for UP and UAs; $V_{4} \sim-300$ $\mathrm{K}$ and $V_{6} \sim-15 \mathrm{~K}$ for ${ }^{23} \mathrm{USb}$. These CF parameters are directly related to $W$ and $x$ [Eq. (10) in Ref. 23]. Since the experimental values of $V_{6}$ are much less than $V_{4}$, we neglect $V_{6}$ and take, as in the equilibrium magnetic structure calculations, ${ }^{30} x=1\left(W=60 B_{4}\right)$. Figure 10 shows the variation of $\kappa_{\|} / \kappa_{\perp}$, evaluated in the vicinity of the ordering temperature $\left(T-T_{N}=0.04 T_{N}\right)$, as a function of $E_{3} / E_{1}$, for the $L-S$ and $j-j$ coupling limits and for the same range of CF parameters as that used in the magnetization calculations. ${ }^{30}$ As can be seen from Fig. $10, \kappa_{\|} / \kappa_{\perp}$ depends strongly on the size and the sign of the CF splitting for the $L-S$ coupling case, and depends rather weakly on the CF splitting in the limit of $j-j$ coupling. (To avoid crowding in the figure, in the $j-j$ limit we have plotted our numerical results only for the two extreme $B_{4}$ values.) Our numerical results show that a positive $B_{4}\left(V_{4}<0\right)$ greatly reduces the relative strength of intra- to interpla-

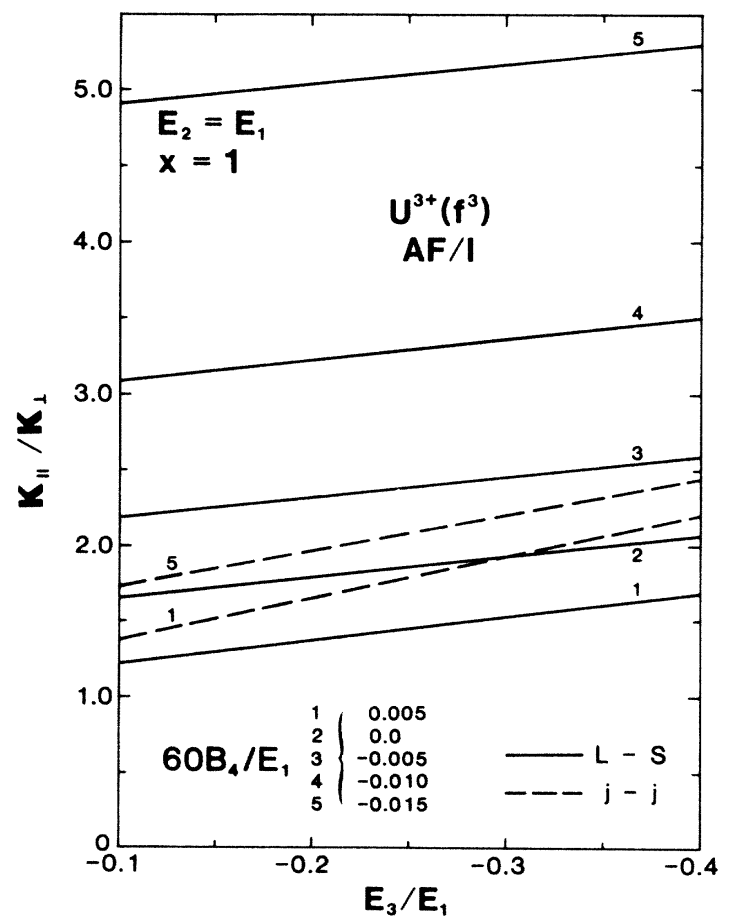

FIG. 10. Ratio of inverse critical correlation lengths parallel and perpendicular to the [001] moment direction for $\mathrm{U}^{3+}\left(f^{3}\right)$ fcc systems as a function of $E_{3} / E_{1}$, for the cases of $L-S$ and $j-j$ coupling and for various values of $C F$ splitting. In all cases the magnetic ordering at $T_{N}$ is antiferromagnetic of type $\mathrm{I}$. (The results for the intermediate values of $B_{4}$ in the $j-j$ limit, interpolate smoothly between the curves labeled 1 and 5.) nar coupling, whereas a negative $B_{4}\left(V_{4}>0\right)$ enhances the anisotropy of $\kappa_{\|} / \kappa_{1}$.

The equilibrium magnetic behavior of USb and UP has been modeled ${ }^{30}$ using ferromagnetic anisotropic two-ion exchange couplings of short range $\left(E_{2}=0\right.$ for USb and $E_{2}=0.09 E_{1}$ for UP) and a moderate antiferromagnetic $H_{1}\left(\sim-0.10 E_{1}\right)$. However, we find that it is not possible to reproduce the experimental values ${ }^{12}$ of $\kappa_{\|} / \kappa_{\perp}$ (our calculations yield $\kappa_{\|}<\kappa_{\perp}$ ) for this range of parameters with short range two-ion interactions. On the other hand, as shown in Fig. 10, systems with longer-range CS-type interactions $\left(E_{1} \sim E_{2}\right)$ favor a cigarlike shape $\left(\kappa_{\|}>\kappa_{\perp}\right)$ critical scattering for a wide range of $E_{3} / E_{1}$ and $\mathrm{CF}$ splitting. For the parameter set $E_{1}=E_{2}=1, E_{3}=-0.1$, $H_{1}=-0.0392, W=-0.1, x=1$, used previously ${ }^{30}$ to model the static magnetic properties of UAs, we find that $\kappa_{||} / \kappa_{\perp}=2.30$ compared to the experimental value ${ }^{12}$ of $3.8 \pm 0.4$. (Upon decreasing the ratio $E_{2} / E_{1}$ to about 0.8 , $\kappa_{\|} / \kappa_{\perp}$ increases to about 3.0.) However, in the limit of such a large CF strength compared to the two-ion interactions, the thermally induced transition between the double- $k$ type-IA and single- $k$ type-I structures was found $^{30}$ difficult to reproduce.

\section{CONCLUSIONS}

The present RPA calculation of the ratio of inverse critical correlation lengths for the longitudinal fluctuations parallel and perpendicular to the moment direction provides further theoretical evidence that the physics of the magnetic behavior of a class of moderately delocalized $\mathrm{Ce}$ and light actinide compounds, including the monopnictides, is indeed due to $f$-electron hybridization with band electrons. In the case of $\mathrm{Ce}^{3+}\left(f^{1}\right)$ systems, our numerical results for $\kappa_{\|} / \kappa_{\perp}$ compare well with those of DCNS experiments. ${ }^{12}$ The reversal of correlation length anisotropy on going to the lighter $\mathrm{Ce}$ monopnictides is found to arise from the increase of the crystal-field splitting $\left(\Gamma_{7}\right.$ low $)$ compared to the hybridization-mediated two-ion interaction.

Although it might superficially appear that there is considerable flexibility in the choice of parameters giving the correlation length behavior, that is not the case. The values of $E_{1}, E_{2}$, and $B_{4}$ are constrained so as to yield the experimentally observed Néel temperature, zero-temperature moment and high (above $T_{N}$ ) temperature crystal-field splitting. Thus in our calculations there is only one adjustable parameter $E_{3}$ to fit the observed ratio $\kappa_{\|} / \kappa_{1}$. Furthermore it should be emphasized that, for $\mathrm{CeBi}$, the $E_{3} / E_{1}=-0.3$ curve in Fig. 5 at the experimental value of $\Delta_{\mathrm{CF}} / k_{B} T_{N}$ uses exactly the parameter values of our previous work ${ }^{26}$ giving agreement with the experimental magnetic excitation spectrum; and these parameter values are in good agreement with the first-principles calculational values $^{43}$ obtained by a synthesis of band and model Hamiltonian theory.

For the case of $\mathrm{Pu}^{3+}\left(f^{5}\right)$ systems, we find the anisotropy in correlation lengths to depend dramatically on the type of intraionic coupling, changing from cigarlike to lenslike shape on going from $j-j$ to $L-S$ (or IC) coupling. It will be interesting to see whether DCNS experiments in 
PuSb will yield a $\kappa_{\|} / \kappa_{\perp}$ of cigar- or lenslike shape. If $\kappa_{\|} / \kappa_{\perp}$ is found experimentally to be greater than unity it would indicate that the behavior of plutonium in the monopnictides is more ceriumlike than our theory presently yields. In that case, we either need to combine the effect of intraionic correlation and of hybridization (with the band electrons) in a physically more accurate way; or since the intermediate coupled wave function used $^{29}$ was not exact, we may need to improve the treatment of the intermediate coupled ground-state wave function in determining the scattering coefficients which arise in our theory. ${ }^{27-29}$ Finally, in considering $\mathrm{U}^{3+}\left(f^{3}\right)$ systems, we find the critical scattering in reciprocal space to be of cigarlike shape $\left(\kappa_{\|}>\kappa_{\perp}\right)$ for both the $L-S$ and $j-j$ coupling limits, and for a wide range of interaction parameters, in qualitative agreement with experiment.

\section{ACKNOWLEDGMENTS}

This research was supported through the National Science Foundation under Grant No. DMR-85-04449. We are grateful to A. Banerjea for valuable discussion of the previously developed theories of the equilibrium and excitation behavior as well as of the present work, and to $\mathbf{A}$. Furrer and B. Hälg for valuable discussion of the critical neutron scattering experiments in the cerium monopnictides.
${ }^{1}$ G. Busch and O. Vogt, Phys. Lett. 25A, 449 (1967); B. R. Cooper, in Magnetism in Metals and Metallic Compounds, edited by J. T. Lopuszanki, A. Pekalski, and J. Przystawa (Plenum, New York, 1976), pp. 225-263.

2J. Rossat-Mignod, P. Burlet, S. Quezel, and O. Vogt, Phys. Scr. 102B, 237 (1980); J. Rossat-Mignod, G. H. Lander, and P. Burlet, in Handbook on the Physics and Chemistry of the Actinides, edited by A. J. Freeman and G. H. Lander (NorthHolland, Amsterdam, 1984), Vol. 1, Chap. 6, pp. 415-512.

${ }^{3}$ P. Burlet, S. Quezel, J. Rossat-Mignod, J. C. Spirlet, J. Rebizant, W. Müller, and O. Vogt, Phys. Rev. B 30, 6660 (1984).

${ }^{4}$ B. R. Cooper, P. Thayamballi, J. C. Spirlet, W. Müller, and O. Vogt, Phys. Rev. Lett. 51, 2418 (1983); K. Mattenberger, O. Vogt, J. C. Spirlet, and J. Rebizant, J. Magn. Magn. Mater. 54-57, 539 (1986).

${ }^{5}$ B. Hälg, A. Furrer, W. Hälg, and O. Vogt, J. Phys. C 14, L961 (1981).

6J. Rossat-Mignod, P. Burlet, J. Villain, H. Bartholin, T. S. Wang, D. Florence, and O. Vogt, Phys. Rev. B 16, 440 (1977).

${ }^{7}$ B. Hälg, A. Furrer, J. K. Kjems, and O. Vogt, Phys. Rev. Lett. 50, 1085 (1983).

${ }^{8}$ P. Burlet, S. Quezel, J. Rossat-Mignod, and O. Vogt, in Proceedings of the Symposium on Neutron Scattering, Berlin, 1984 (unpublished).

${ }^{9}$ B. R. Cooper and O. Vogt, J. (Paris) Colloq. Phys. 32, C1-1026 (1971); B. R. Cooper, M. Landolt, and O. Vogt, in Proceedings of the International Conference on Magnetism-Moscow, edited by R. P. Ozerov and Yu. A. Izyumov (Nauka, Moscow, 1974), Vol. 5, pp. 354-360.

${ }^{10}$ B. R. Cooper and R. Siemann, J. Appl. Phys. 50, 1991 (1979); R. Siemann and B. R. Cooper, Phys. Rev. Lett. 44, 1015 (1980); J. Magn. Magn. Mater. 15-18, 573 (1980).

${ }^{11}$ B. Coqblin and J. R. Schrieffer, Phys. Rev. 185, 847 (1969); B. Cornut and B. Coqblin, Phys. Rev. B 5, 4541 (1972).

${ }^{12}$ B. Hälg and A. Furrer, J. Appl. Phys. 55, 1860 (1984).

${ }^{13}$ B. Hälg, A. Furrer, W. Hälg, and O. Vogt, J. Magn. Magn. Mater. 29, 151 (1982).

${ }^{14}$ B. Hälg, A. Furrer, W. Hälg, and O. Vogt, J. Appl. Phys. 53, 1927 (1982).

${ }^{15}$ G. H. Lander, S. K. Sinha, D. M. Sparlin, and O. Vogt, Phys. Rev. Lett. 40, 523 (1978).

${ }^{16}$ S. K. Sinha, G. H. Lander, S. M. Shapiro, and O. Vogt, Phys. Rev. Lett. 45, 1028 (1980); Phys. Rev. B 23, 4556 (1981).
Rev. Lett. 45, 1028 (1980); Phys. Rev. B 23, 4556 (1981).

${ }^{17}$ T. M. Holden, W. J. L. Buyers, E. C. Svensson, and G. H. Lander, Phys. Rev. 26, 6227 (1982).

${ }^{18}$ R. J. Birgeneau, E. Bucher, J. P. Maita, L. Passell, and K. C. Turberfield, Phys. Rev. B 8, 5345 (1973).

${ }^{19}$ H. Takahashi, K. Takegahara, A. Yanase, and T. Kasuya, in Valence Instabilities, edited by P. Wachter and H. Boppart (North-Holland, Amsterdam, 1982), pp. 379-382.

${ }^{20}$ P. Thayamballi and B. R. Cooper, Phys. Rev. B 30, 2931 (1984).

${ }^{21}$ J. M. Wills, B. R. Cooper, and P. Thayamballi, J. Appl. Phys. 57, 3185 (1985).

${ }^{22}$ R. Troc and D. J. Lam, Phys. Status Solidi B 65, 317 (1974).

${ }^{23}$ G. H. Lander, M. H. Mueller, D. M. Sparlin, and O. Vogt, Phys. Rev. B 14, 5035 (1976).

${ }^{24}$ B. R. Cooper, J. Magn. Magn. Mater. 29, 230 (1982); David Yang and Bernard R. Cooper, J. Appl. Phys. 53, 1988 (1982).

${ }^{25}$ B. R. Cooper, P. Thayamballi, and D. Yang, J. Appl. Phys. 55, 1866 (1984); P. Thayamballi, D. Yang, and B. R. Cooper, Phys. Rev. B 29, 4049 (1984).

${ }^{26}$ P. Thayamballi and B. R. Coooper, Phys. Rev. B 31, 5911 (1985).

${ }^{27}$ P. Thayamballi and B. R. Coooper, J. Appl. Phys. 53, 7902 (1982).

28P. Thayamballi and B. R. Coooper, J. Appl. Phys. 55, 1829 (1984).

${ }^{29}$ A. Banerjea, B. R. Cooper, and P. Thayamballi, Phys. Rev. B 30, 2671 (1984).

${ }^{30}$ P. Thayamballi and B. R. Cooper, Phys. Rev. B 31, 6004 (1985).

${ }^{31}$ For a review as of mid-1984 see B. R. Cooper, R. Siemann, D. Yang, P. Thayamballi, and A. Banerjea, in Handbook on the Physics and Chemistry of the Actinides, edited by A. J. Freeman and G. H. Lander (North-Holland, Amsterdam, 1985), 32P. W. Anderson, Phys. Rev. 124, 41 (1961).

33J. R. Schrieffer and P. A. Wolff, Phys. Rev. 149, 491 (1966); J. R. Schrieffer, J. Appl. Phys. 38, 1143 (1967).

${ }^{34}$ M. A. Ruderman and C. Kittel, Phys. Rev. 96, 99 (1954); T. Kasuya, Progr. Theor. Phys. 16, 45 (1956); 16, 58 (1956); K. Yosida, Phys. Rev. 106, 893 (1957).

${ }^{35}$ D. Yang and B. R. Cooper, J. Appl. Phys. 53, 1988 (1982).

${ }^{36}$ S. B. Haley and P. Erdos, Phys. Rev. B 5, 1106 (1972); D. H. Yang and Y. L. Wang, ibid. 10, 4714 (1974). 
${ }^{37}$ M. Tinkham, Group Theory and Quantum Mechanics (McGraw-Hill, New York, 1964).

${ }^{38}$ K. R. Lea, M. J. M. Leask, and W. P. Wolf, J. Phys. Chem. Solids 23, 1381 (1962).

${ }^{39}$ K. W. H. Stevens, Proc. Phys. Soc. London Sect. A 65, 209 (1952).

${ }^{40}$ L. S. Ornstein and F. Zernike, Proc. Acad. Sci. (Amsterdam)
17, 793 (1914); Phys. Z 19, 134 (1918).

${ }^{41}$ S. K. Ma, in Modern Theory of Critical Phenonema, edited by D. Pines (Benjamin, New York, 1976), pp. 104-105.

${ }^{42}$ G. H. Lander, A. Delapalme, P. J. Brown, J. C. Spirlet, J. Rebizant, and O. Vogt, Phys. Rev. Lett. 53, 2262 (1984).

43J. M. Wills and B. R. Cooper, J. Magn. Magn. Mater. 54-57, 1049 (1986) 